\title{
SYSTEMATIC CHECKLIST WITH A REVIEW OF PUBLICATIONS \\ OF FAMILY HALICTIDAE OF EGYPT (ORDER: HYMENOPTERA) \\ 2- SUBFAMILIES: NOMIOIDINAE, NOMIINAE AND ROPHITINAE
}

\author{
EL-AKKAD, M. K. and E. EL. A. M. BIBARS \\ Plant Protection Research Institute, ARC, Dokki, Giza, Egypt \\ (Manuscript received 12 February 2015)

\begin{abstract}
$\mathrm{T}$ his is the second paper of the systematic checklist of the Egyptian species of family Halictidae (Order: Hymenoptera), includes subfamilies Nomioidinae, Nomiinae and Rophitinae is presented. The subfamily Nomioidinae consistingof 11 species belonging to 2 genera and 2 subgenera. Three species of this subfamily were added to the Egyptian fauna. The subfamily Nomiinae includes 8 species belonging to 3 genera and 2 subgenera. Two species of this subfamily were added to Egyptian genera. In addition to three species were recorded from neighboring countries and probably will be recorded later from Egypt. The checklist is arranged according to species membership in higher - level taxa, based on the most recent literature of family Halictidae.
\end{abstract} \\ fauna. The third subfamily includes 6 species belonging to 3
}

\section{INTRODUCTION}

Family Halictidae includes four subfamilies namely: Halictinae, Nomioidinae, Nomiinae and Rophitinae (Michener, 2007), it contains about 72 genera and nearly 3500 currently recognized species worldwide (Pesenko, 2007). The Egyptian fauna and also Palaearctic fauna of subfamilies Nomioidinae, Nomiinae and Rophitinae were studied by several scholars such as: Handlirsch (1888); Friese (1898); Debski (1917); Alfken (1927); Blüthgen (1925, 1933 \& 1934); Warncke (1976, 1979 \& 1980); Pesenko (1983, 2000 \& 2007); Ebmer (1984, 1993 \& 1999); Pauly (1990 \& 2012); ElAkkad (1993); Baker (1996 \& 2002); Shoukry, et al. (2004); Pesenko \& Pauly (2005 \& 2009); Michener (2007).

The present study is carried out to clarify the recent taxonomic status of three subfamilies of family Halictidae and their members in Egypt. 


\section{MATERIALS AND METHODS}

The checklist of family Halictidae of Egypt is based on all records found in the available entomological literature and those reviewed from the preserved specimens in the main Egyptian insect collections. These collections are: the Egyptian Entomological Society collection, Ain Shams University collection and collection of the Plant Protection Research Institute, Ministry of Agriculture. Identification of the specimens had been confirmed early by A. W. Ebmer (Austria) and the late Prof. Dr. K. Warncke (Germany), the specialists of Halictidae of Palaearctic region. These specimens preserved in the collection of Plant Protection Research Institute, Ministry of Agriculture. The nomenclature and taxonomic status are adopted from the following sources: Pesenko (1984); Warncke (1992); Ebmer (1976 \& 1995); Michener (2007), Bogusch \& Straka (2012), Pauly's Atlas Hymenoptera online (2012) and Ascher \& Pickering (2014). The checklist is arranged according to species membership in higher - level taxa, based on the most recent literature. Synonyms, taxonomic notes and published records from Egypt are included.

\section{Family: Halictidae}

\section{Subfamily: Nomioidinae Börner, 1919}

Nomioidinae Börner, 1919, Biologisches Zentralblatt, 39: 145-185.

The subfamily Nomioidinae consists of minute species $(2.5-6.5 \mathrm{~mm}$.) often with yellow or cream markings on head, mesosoma and metasoma. It includes three genera all over the world; two of them are represented in Egypt.

\section{Genus: Ceylalictus Strand, 1913}

This genus is represented by a single subgenus covering only two species in Egypt as follows:

Subgenus: Ceylalictus Strand, 1913, Arch. Naturg., 79 (12): 127 - 171.

\section{9: Ceylalictus (Ceylalictus) punjabensis (Cameron, 1907)}

= Ceratina punjabensis Cameron, 1907, Journal of the Bombay Natural History Society, 17 (4): 1003.

\section{Published records from Egypt:}

Debski (1917): 31 - 32 (key to males and females); 46 - 50 (Re- description): Nomioides fasciatus Friese. Blüthgen (1925): 61: Nomioides fasciata var. punjabensis Blüthgen. Alfken (1927): 105: Nomioides fasciata Friese. Blüthgen (1933): 24: Nomioides fasciata Friese. El-zoheiry \& Mohamed (1949): 75: Nomioides fasciata Friese. Shalaby (1958): 80: Nomioides fasciata Friese. Pesenko (1983): 178: (key to females); 179 (key to males): Ceylalictus (Ceylalictus) punjabensis (Cameron). ElAkkad (1993): 174 - 175 (key to females); 175 - 176 (key to males); 185- 188 
(Synonym, re - description; 54 specimens examined $(24 q q+30 \hat{\jmath})$ ): Nomioides fasciata Friese. Pauly et al. (2002), Bulletin de I'Institut Royal des Sciences Naturelles de Belgique, Entomologie, 72: 206: (Synonymy \& distribution): Ceylalictus (Ceylalictus) punjabensis (Cameron). Pesenko \& Pauly (2005): 143 - 144 (key); 157 160 (Re- description): Ceylalictus (Ceylalictus) punjabensis (Cameron). Pesenko \& Pauly (2009): 219: Ceylalictus (Ceylalictus) punjabensis (Cameron). Saini \& Vikram (2012): 137 (Distribution): Ceylalictus (Ceylalictus) punjabensis (Cameron).

\section{0: Ceylalictus (Ceylalictus) variegatus (Olivier, 1789)}

= Apis variegata Olivier, 1789: Encyclopedia méthodique Paris: 139.

\section{Published records from Egypt:}

Handlirsch (1888): 402 - 404: Nomioides variegate (Olivier). Friese (1898): 307: Original description of Nomioides fasciatus sp. n. ㅇ, $\hat{\jmath}$, [Lectotype: Heluan, Egypt]. Debski (1917): 31 \& 33 (key to males and females); 46 - 50 (Re- description): Nomioides variegates (Olivier). Sowerby (1917), Entomologist, 50 (2): 31: Nomioides variegate (Olivier). Alfken (1927): 105: Nomioides variegate (Olivier). Blüthgen (1933): 24: Nomioides variegate (Olivier). El-zoheiry \& Mohamed (1949): 75: Nomioides variegate (Olivier). Shalaby (1958): 81 : Nomioides variegate Olivier. Pesenko (1983): 178 (key to females); 179 (key to males): Ceylalictus (Ceylalictus) variegatus (Olivier). El-Akkad (1993): 174 - 175 (key to females); 175 - 176 (key to males); 198-202 (Synonymy, re - description; 375 specimens examined (145웅 +

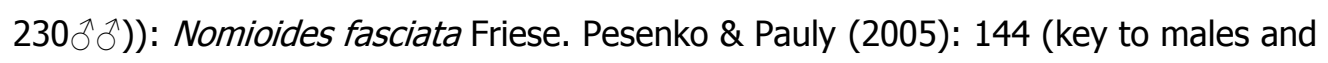
females); 160 - 164 (Re- description): Ceylalictus (Ceylalictus) variegatus (Olivier). Pesenko \& Pauly (2009): 219 - 221: Ceylalictus (Ceylalictus) variegatus (Olivier). Saini \& Vikram (2012): 137 (Distribution): Ceylalictus (Ceylalictus) variegatus (Olivier).

\section{Genus Nomioides Schenck 1867}

Nomioides Schenck, 1867, Berliner Entomologische Zeitschrift 10: 333.

This genus is widespread in the Palaearctic region. It is represented by a single subgenus covering nine species from Egypt; three of them newly added to the Egyptian fauna namely: N. (Nomioides) bluethgeni Pesenko, N. (Nomioides) micheneri Pesenko \& Pauly and $N$. (Nomioides) ornatus Pesenko.

Subgenus Nomioides Schenck, 1867, Berliner Entomologische Zeitschrift 10: 333

\section{1- Nomioides (Nomioides) aegyptiaca Blüthgen, 1933}

\section{Published records from Egypt:}

Blüthgen, (1933): 25 - 27: Original Description of Nomioides aegyptiaca sp. n. (Type: 1 우 from Gabal el Asfer 27/10/1925 \& 1 ^ from Wadi Nesour (Heluan), 23/10/1925). El-zoheiry \& Mohamed (1949): 75: Nomioides aegyptiaca Blüthgen. Shalaby (1958): 
80: Nomioides aegyptiaca Blüthgen. El-Akkad (1993): 174 - 175 (key to females); 175 - 176 (key to males); 177-179 (Synonymy, re - description; Holotype examined ( $q$ + §)): Nomioides aegyptiaca Blüthgen. Pesenko \& Pauly (2005): 133 \& 215: They could not examined the type of this species, for this reason, they considered Nomioides aegyptiaca as a dubious name belong to genus Nomioides subgenus Nomioides until examination of the type.

\section{2- Nomioides (Nomioides) bluethgeni Pesenko, 1979}

$=$ Nomioides (Nomioides) bluethgeni Pesenko 1979, Trudy Vsesoyuznogo Entomologicheskogo Obshchestva, 61:176 0 .

= Nomioides (Nomioides) bluethgeni Pesenko, 1983: 125ㅇ.

\section{Published records from Egypt:}

Pesenko (1979): 176: Original description of Nomioides (Nomioides) bluethgeni sp. n. Pesenko (1983): 125 (key to females); 128 (key to males): Nomioides (Nomioides) bluethgeni Pesenko. Pesenko \& Pauly (2005): 168 - 172 (key to males and females); 174 (Synonymy \& re- description): Nomioides (Nomioides) bluethgeni Pesenko. Pauly (2012): (Distribution: Morocco, Egypt, Kenya, Jordon, Syria and Armenia): Nomioides (Nomioides) bluethgeni Pesenko.

\section{3- Nomioides (Nomioides) deceptor deceptor Saunders, 1908}

= Nomioides deceptor Saunders 1908, Trans. Royal Entom. Soc. London, (2): 223, ㅇ, ô.

\section{Published records from Egypt:}

Blüthgen, (1925): 11: Nomioides minutissima var. deserticola Blüthgen [Holotype $\delta_{\text {, }}$ Khanka, 25.V.1914, Egypt [In Coll. Ministry Agric., Cairo]. Blüthgen (1933): 23: Nomioides deceptrix Saunders: Wadi Um Elek. Blüthgen (1934): 192: Nomioides deceptrix Saunders: Wadi Um Elek. El-zoheiry \& Mohamed (1949): 75: Nomioides minutissimus K; Nomioides minutissima var. deserticola Blüthgen and Nomioides minutissima var. nubica Blüthgen. Shalaby (1958): 80: Nomioides deceptor S. \& Nomioides minutissima K. Pesenko (1983): 138: Nomioides minutissima deceptor Saunders. El-Akkad (1993): 174 - 175 (key to females); 175 - 176 (key to males); 180-182 (Synonym, re - description; 8 specimens examined $\left(5\right.$ 우 $\left.+3 \hat{\delta}^{\widehat{\jmath}}\right)$ ): Nomioides deceptor Saunders. Pesenko \& Pauly (2005): 168 - 172 (key to males and females); 174 (Synonymy \& re- description): Nomioides (Nomioides) deceptor deceptor Saunders. Pesenko \& Pauly (2009): 223 - 224: Nomioides (Nomioides) deceptor deceptor Saunders. Pauly (2012): (Distribution: North Africa and Arabian Peninsula): Nomioides (Nomioides) deceptor deceptor Saunders.

\section{4- Nomioides (Nomioides) elbanus Blüthgen, 1934}

= Nomioides elbana Blüthgen 1934: 200. 


\section{Male: unknown}

\section{Published records from Egypt:}

Blüthgen (1934): 192 \& 200: Original description of Nomioides elbana sp. n. (Holotype: , Gebel Elba: 21.1.1933, Egypt; in Coll. Ministry Agric., Cairo). El-Akkad (1993): 174 - 175 (key to females); 183-184 (Synonym, re - description; Type examined (2 우): Nomioides deceptor Saunders. Pesenko \& Pauly (2005): 172 (key to females); 178 (Synonymy \& re- description): Nomioides (Nomioides) elbanus Blüthgen. Pesenko \& Pauly (2009): 224: Nomioides (Nomioides) elbanus Blüthgen. Pauly (2012): (Distribution: Algeria, Egypt and Arabian Peninsula): Nomioides (Nomioides) elbanus Blüthgen.

\section{5- Nomioides (Nomioides) micheneri Pesenko \& Pauly, 2005}

$=$ Nomioides (Nomioides) micheneri Pesenko \& Pauly 2005: 191-193, ㅇ, $\hat{0}$.

\section{Published records from Egypt:}

Pesenko \& Pauly (2005): 171 - 172 (key to males and females); 191 - 193 (Original description of male and female; Paratypes (294 specimens): Egypt: Sinai, Wadi Suor, 3/5/1993): Nomioides (Nomioides) micheneri Pesenko \& Pauly. Pesenko \& Pauly (2009): 225: Nomioides (Nomioides) micheneri Pesenko \& Pauly.

\section{6- Nomioides (Nomioides) ornatus Pesenko, 1983}

$=$ Nomioides (Nomioides) ornatus Pesenko 1983: $124 \& 127 ;$;, 0 .

\section{Published records from Egypt:}

This species recorded from Egypt (Pesenko, 1983: 160), one male specimen from Fayed, V/ 1943, Coll. Priesner (Paratype).

Pesenko \& Pauly (2005): 168 - 172 (key to males and females); 195 - 196 (Synonymy \& re- description): Nomioides (Nomioides) ornatus Pesenko. Pesenko \& Pauly (2009): 225: Nomioides (Nomioides) ornatus Pesenko.

Pauly (2012): (Distribution: Egypt, Niger, Burkinafaso, Chad, Israel and Kasakhstan): Nomioides (Nomioides) ornatus Pesenko.

\section{7- Nomioides (Nomioides) rotundiceps Handlirsch, 1888}

= Nomioides rotundiceps Handlirsch 1888: 405, Syntypes: 3 +, , Egypt, Cairo.

\section{Published records from Egypt:}

Handlirsch (1888): 405: Original description of female of Nomioides rotundiceps sp. $\mathrm{n}$. Alfken (1927): 105: Nomioides rotundiceps Handlirsch. Blüthgen (1933): 24: Nomioides rotundiceps Handlirsch. El-zoheiry \& Mohamed (1949): 75: Nomioides rotundiceps var. viridanus Bl. Shalaby (1958):81: Nomioides rotundiceps \& Nomioides rotundiceps var. viridanus Bl. Pesenko (1983): 124 (key to females); 127 (key to males): Nomioides (Nomioides) rotundiceps Handlirsch. El-Akkad (1993): 174 - 175 (key to females); 175 - 176 (key to males); 192-194 (Synonym, re - description; 63 
specimens examined $(19+9$ 우 $+44 \hat{\jmath} \widehat{\jmath}))$ : Nomioides rotundiceps Handlirsch. Pesenko \& Pauly (2005): 170 - 172 (key to males and females); 197 - 199 (re- description \& distribution): Nomioides (Nomioides) rotundiceps Handlirsch. Pesenko \& Pauly (2009): 226 - 227: Nomioides (Nomioides) rotundiceps Handlirsch. Pauly (2012): (Distribution: Morocco, Algeria, Egypt, Mauritania, Senegal, Mali, Burkina Faso, Niger, Cameroon, Chad, Sudan, Kenya, Israel, Jordon, Arabian Peninsula, southern Iran): Nomioides (Nomioides) rotundiceps Handlirsch.

\section{8- Nomioides (Nomioides) squamiger Saunders, 1908}

= Nomioides squamiger Saunders 1908, Trans. Royal Entom. Soc. London, (2): 222, ㅇ, $\hat{0}$.

\section{Published records from Egypt:}

Debski (1917): 31 (Key to males and females); 35 (Nomioides sp.?): Nomioides squamiger Saunders. Blüthgen (1934): 201 table to comparing between females of Nomioides squamiger, N. curvilineata and N. elbana. El-Akkad (1993): 174 - 175 (key to females); 175 - 176 (key to males); 189-191 (Synonymy, re - description; 18 specimens examined $(16+90+2 \widehat{\jmath} \widehat{\partial}))$ : Nomioides minutissima Rossi. Pesenko \& Pauly (2005): 168 - 172 (key to males and females); 199 - 201 (Synonymy, re-description \& distribution [Egypt: Sinai: Wadi Sour; 3/5/1993]): Nomioides (Nomioides) squamiger Saunders. Pauly (2012): (Distribution: North Africa, Israel, Arabian Peninsula): Nomioides (Nomioides) squamiger Saunders.

\section{9- Nomioides (Nomioides) turanicus Morawitz, 1876}

= Nomioides turanica Morawitz 1876, Turkestan Mellifera: 214, , 0 .

\section{Published records from Egypt:}

Handlirsch (1888): 402: Nomioides turanica Morawitz. Debski (1917): 31 (Key to males); 32 (key to females); 33 - 35: Original description of Nomioides heluanensis [Holotype $\delta$ : Egypt, Heluan 4/9 /1916] \& Nomioides storeyi [Holotype o: Egypt, Qaliub 9/7/1912]. Alfken (1927): 105: Nomioides turanica Morawitz. Blüthgen (1925): 20 Nomioides turanica var. nubica Blüthgen. Blüthgen (1933): 23: Nomioides turanica Morawitz. Blüthgen (1934): 192: Nomioides turanica var. subvariegata Blüthgen [Holotype: †, Djebel Elba (Wadi Aideb); Egypt]. El-zoheiry \&Mohamed (1949): 75: Nomioides storeyi Debski \& Nomioides turanica Morawitz. Shalaby (1958): 81: Nomioides storeyi Debski \& Nomioides turanica Morawitz. Pesenko (1983): 125 (key to females); 130 (key to males): Nomioides turanica Morawitz. El-Akkad (1993): 174 - 175 (key to females); 175 - 176 (key to males); 195-197 (Synonymy, re - description; 20 specimens examined $\left(11+q\right.$ + $+9 \hat{\delta}^{\widehat{\jmath}}$ )): Nomioides turanica Morawitz. Pesenko \& Pauly (2005): 168, 171 (key); 201 - 203 (re- description \& distribution): Nomioides (Nomioides) turanica Morawitz. Pesenko 
\& Pauly (2009): 234: Nomioides (Nomioides) turanica Morawitz. Pauly (2012): (Distribution: North Africa to Sudan, Djibouti, Senegal, and Mauritania in the south; deserts of western Asia to Kirghizia and Pakistan in the east): Nomioides (Nomioides) turanica Morawitz.

\section{Subfamily: Nomiinae}

This subfamily is revised by Shoukry et al. (2004). Three genera belonging to this subfamily are represented in Egypt. Two species were added to the Egyptian fauna by Warncke (1980) and Baker (2002).

\section{Genus: Leuconomia Pauly, 1980}

Genus: Leuconomia Pauly, 1980, Revue de Zoologie Africaine, 94: 124.

= Paranomina Michener, 1944, Bull. Amer. Mus. Nat. History, 130: 251

\section{0- Leuconomia Iutea (Warncke, 1976)}

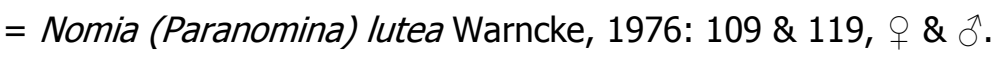

\section{Published records from Egypt:}

Warncke (1976): 94 (key to females); 96 (key to males); 109 (Distribution) \& 119 (Original description: Holotype: +, Egypt, Heluan, 21. 10. 1930): [Nomia

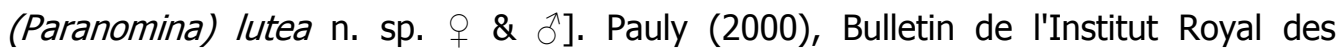
Sciences Naturelles de Belgique, Entomologie, 70: 167, 180 - 181 (Synonymy \& Distribution): Leuconomia lutea (Warncke). Shoukry et al. (2004): 146 - 147 (Redescription): Nomia (Leuconomia) Iutea Warncke. Pauly (2012): Leuconomia lutea (Warncke).

\section{Genus: Nomiapis Cockerell, 1919}

Nomia (Nomiapis) Cockerell, 1919, Proceedings of the United States Nat. Museum, 55: 208.

51- Nomiapis bispinosa (Brulle, 1832)

Nomia bispinosa Brulle, 1832, Expédition scientifique de Morée: 348, $\widehat{0}$. Nomia rufiventris Spinola, 1839, Ann. Soc. Entom. France, 7: 514: Nomia ruficornis Spinola, 1839, Ann. Soc. Entom. France, 7: 514; đ̇; (Egypt)

\section{Published records from Egypt:}

Alfken (1927): 103: Nomia rufiventris Spin. El-zoheiry \& Mohamed (1949): 76: Nomia rufiventris Spin \& Nomia rufiventris var. albocincta Luc. Shalaby (1958): 80: Nomia rufiventris Spin. Warncke (1976): 94 - 96 (key to females); 96 - 99 (key to males); 109 (Synonymy \& distribution): Nomia (Nomiapis) unidentata Olivier. Warncke (1980): 264, 269: Nomia (Nomiapis) unidentata Olivier. Baker (2002): 34 - 35 (Synonymy); 50 - 58 (key to males): Nomiapis (Nomiapis) bispinosa (Brulle). Shoukry et al. (2004): 149 (key to males and females); 153- 154 (Synonymy \& re- description): Pseudapis 
(Pseudapis) unidentata (Olivier). Saini \& Vikram (2012): 148 (Synonymy \& distribution): Nomiapis bispinosa (Brulle).

\section{Genus Pseudapis Kirby, 1900}

Subgenus: Pseudapis Kirby, 1900, Bulletin of the Liverpool Museums, 3: 15.

$$
\text { = Nomia (Lobonomia) Warncke, 1976: } 99 .
$$

\section{2- Pseudapis (Pseudapis) anomala Kirby, 1900}

Pseudapis anomala Kirby, 1900, Bulletin of the Liverpool Museums, 3: 16: $\hat{\partial}$.

\section{Published records from Egypt:}

Warncke (1980): 366 (key to males); 367 (key to females); 368 (Original description: Holotype: $\delta$ Gebel Elba, Egypt: Paratype: 2 우, Gebel Elba, Wadi Aideb, 4. 2. 1933): Nomia (Pseudapis) sudanica n. sp. Baker (2002): 29: (Synonymy); 50 - 58 (key to males): Pseudapis (Pseudapis) anomala Kirby.

\section{3- Pseudapis (Pseudapis) dixica (Warncke, 1976)}

Nomia (Lobonomia) dixica Warncke, 1976: 117 - 118, figs. 9, 30; ㅇ, ở;

\section{Published records from Egypt:}

Warncke (1976): 94- 96 (key to females); 96 - 99 (key to males); 117 - 118 (Original description: Holotype: $\uparrow$, Ain Sefra, Algeria): Nomia (Lobonomia) dixica sp. n. Pauly (1990): 68 (key to males); 72 (Diagnosis): Pseudapis dixica (Warncke). Baker (2002): 33: (Synonymy); 50 - 58 (key to males): Pseudapis (Pseudapis) dixica (Warncke). Shoukry et al. (2004): 149 (key to males and females); 151 (Redescription): Pseudapis (Pseudapis) dixica (Warncke).

\section{4- Pseudapis (Pseudapis) fayumensis Baker, 2002}

Pseudapis (Pseudapis) fayumensis Baker, 2002: 39- 42; Holotype $\delta$ : Fayoum, Karanis, 15.6.1990.

\section{Published Records from Egypt:}

Baker (2002): 39 - 42 (Original description: Holotype: ${ }^{\Uparrow}$ Egypt, Fayoum, Karanis, 15. 6. 1990 [deposited in Oxford University Museum of Natural History, Oxford]; 50 - 58 (key to males); male genitalia figure 13: Pseudapis (Pseudapis) fayumensis $\mathrm{sp} . \mathrm{n}$.

\section{5- Pseudapis (Pseudapis) innesi (Gribodo, 1894)}

Nomia innesi Gribodo, 1894, Bollettino della Società Entomologica Italiana [Firenze] 26: 126, ふै; Egypt.

\section{Published records from Egypt:}

Alfken (1927): 103: Nomia edentate Morawitz. El-zoheiry \& Mohamed (1949): 76: Nomia edentata Morawitz \& Nomia innesi Gribodo. Shalaby (1958): 80: Nomia innesi Gribodo \& Nomia edentate Morawitz. Warncke (1976): 94 - 96 (key to females); 96 99 (key to males); 99 (Synonymy \& distribution): Nomia (Lobonomia) innesi Gribodo. Pauly (1990):67 - 68 (key to males and females); 73 (Diagnosis, distribution): 
Pseudapis innesi (Gribodo). Baker (2002): 28: (Synonymy); 50 - 58 (key to males): Pseudapis (Pseudapis) innesi (Gribodo). Shoukry et al. (2004): 148 - 149 (key to males and females); 152 (Re- description): Pseudapis (Pseudapis) innesi (Gribodo).

\section{6- Pseudapis (Pseudapis) nilotica (Smith, 1875)}

$=$ Nomia nilotica Smith, 1875, Trans. Entom. Soc. London: 63, +; White Nile

\section{Published records from Egypt:}

Alfken (1927): 103: Nomia magrettii Gribodo. Warncke (1976): 94 - 96 (key to females); 96 - 99 (key to males); 105 (Synonymy \& distribution): Nomia (Lobonomia) armata Olivier. Warncke (1980): 363, 365: Nomia (Lobonomia) armata ssp. latipes Morawitz. Pauly (1990): 68 (key to males and females); 70 (Synonymy \& Distribution): Pseudapis armata (Olivier). Baker (2002): 22 - 24 (Synonymy); 50 58 (key to males): Pseudapis (Pseudapis) nilotica (Smith). Shoukry et al. (2004): 149 (key to males and females); 149 - 150 (Re- description): Pseudapis (Pseudapis) armata (Olivier).

\section{7- Pseudapis (Pseudapis) nubica (Warncke, 1976)}

Nomia (Lobonomia) nubica Warncke, 1976: 117.

\section{Published Records from Egypt:}

Warncke (1976): 94 - 96 (key to females); 117 (Original description of female: Holotype: + Helioplis, IX. 1913; Egypt): Nomia (Lobonomia) nubica sp. n. Pauly (1990):67 - 68 (key to males and females); 76 (Diagnosis of male and female \& Distribution: Egypt and Somale): Pseudapis nubica (Warncke). Baker (2002): 33: (Synonymy); 50 - 58 (key to males): Pseudapis (Pseudapis) nubica (Warncke). Shoukry et al. (2004): 149 (key to females); 153 (Re- description): Pseudapis (Pseudapis) nubica (Warncke).

\section{Subfamily: Rophitinae (= Dufoureinae)}

The subfamily Rophitinae needs further taxonomic studies; where the number of recorded species from Egypt very few compared to the number of recorded species from Palaearctic Region. It is recognized by the following characters: antennal sockets are below the mid-length of eyes and somewhat closer to the upper clypeal margin. Anterior margin of labrum is rounded or sometimes truncate and the hind basitarsus without penicillus. Only three genera and 6 species are recorded from Egypt.

\section{Genus: Dufourea Lepeletier, 1841}

Subgenus Dufourea Lepeletier, 1841, Hist. Nat. Insects Hymen. Vol. 2: 227 


\section{8- Dufourea (Dufourea) ciliata Ebmer, 1993}

\section{Published records from Egypt:}

\section{Female unknown}

Ebmer (1993): 24 - 25 (Original description: Holotype: Egypt, Kom Ombo, 16 18/2/1958 and Paratype: Egypt, Wadi Katara, 3. 1988): Dufourea (Dufourea) ciliata sp. n.; O. Pauly (2012): (Distribution: Egpt): Dufourea (Dufourea) cliata Ebmer

\section{9- Dufourea (Dufourea) rufiventris Friese, 1898}

\section{Published Records from Egypt:}

Friese (1898): 303-313 (Original description: Egypt): Dufourea rufiventris sp. n. Elzoheiry \& Mohamed (1949): 76: Dufourea rufiventris Friese Shalaby (1958): 39: Dufourea rufiventris Friese. Ebmer (1984), Sencken bergiana biol., 64: 356: Dufourea (Dufourea) rufiventris Friese. Ebmer (1999): 198 (Distribution: Egypt and Israel: Dufourea (Dufourea) rufiventris Friese. Pauly (2012): (Distribution: Egypt and Israel): Dufourea (Dufourea) rufiventris Friese.

\section{0- Dufourea (Dufourea) similis Friese, 1898}

\section{Published records from Egypt:}

Friese (1898): 303 - 313: Original description of Dufourea similis sp. n. (Egypt).

El- zoheiry \& Mohamed (1949): 76: Dufourea similis Friese. Shalaby (1958): 39: Dufourea similis Friese. Ebmer (1999): 186 (Distribution: Tunisa, Moracco, Libya, Egypt, Israel and Syria): Dufourea (Dufourea) similis Friese. Pauly (2012): Dufourea (Dufourea) similes Friese.

Subgenus: Rophites Spinola, 1808, Inectorum Liguriae species Novae aut Rariors: $2 \& 72$.

\section{1- Rophites quadratus Friese, 1898}

\section{Published records from Egypt:}

Friese (1898): 305 (Original description: Egypt): Rophites quadrates sp. n. El- zoheiry \& Mohamed (1949): 76: Rophites quadrates Friese. Shalaby (1958): 39: Rophites quadrates Friese.

Michener (2007): 437: Pararhophites quadratus Friese.

The systematic postion of this species as follows:

Family Megachilidae: Subfamily Pararhophitinae: tribe Pararhophitini.

\section{Genus: Systropha Illiger, 1806}

Subgenus: Systropha Illiger, 1806, Magazin für Insektenkunde, 5: 145

\section{2- Systropha (Systropha) hirsute Spinola, 1839}

= Systropha hirsuta Spinola, 1839, Annales de la Société Entom. France, 7: 516, Egypt, Nobia or Arabia. Lectotype $\widehat{\delta}$ from Egypt preserved in Museo Regionale di Storia Naturale, Torino, Italy. 


\section{Published records from Egypt:}

Casolari \& Casolari Moreno (1980), Museo Regionale di Scienze Naturali: 159 (Distribution: Egypt): Systropha pilosa Spinola. Warncke (1992), Linzer biologische Beiträge, 24: 742: Original description of Systropha christae sp.n. from Israel. Baker

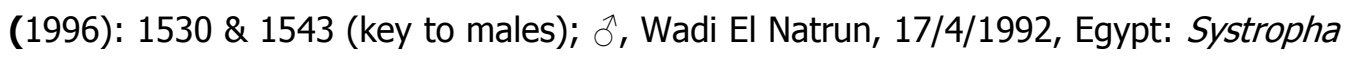
hirsuta Spinola. (= Systropha christae Warncke, 1992) Patiny \& Michez(2006), Annales de la Société entomologique de France, 42 (1):30 \& 31 (key to males); 33 (key to females): Systropha (Systropha) hirsuta Spinola.

\section{Genus: Trilia Vachal}

\section{3- TRILIA MUOTI VACHAL, 1900}

=Dufourea (Trilia) muoti Vachal, 1900, Ann. Soc. Entom. France, 68: 534. $\hat{\sigma}$,

\section{Published Records from Egypt:}

Warncke (1979): 118: Rophites muoti Vachal. Ebmer (2008), Linzer Biologische Beitrage, 40 (1): 605: Trilia muoti Vachal; ( $\%$, Lectotype from Algeria: Ghardaia). Astafurova (2010), Entomological Review, 90 (6): 760 - 763: (key to species and distribution: Algeria, Tunisia and Egypt)

\section{Additional species can be expected later in Egypt}

The following species recorded from neighboring countries and probably will be recorded later from Egypt:

\section{1- Dufourea (Dufourea) chagrina (Warncke, 1979)}

\section{Published records from neighboring countries:}

Warncke (1979): 111- 155: Original description and distribution (Morocco, Tunisia and Israel):Dufoura chagrinachagrina sp. $n$. Ebmer (1993): 20: (Distribution: Tunisia and Israel): Dufourea (Dufourea) chagrina (Warncke). Ebmer (1999): 187: (Diagnosis and Distribution: Israel, Tunisia and Morocco): Dufourea (Dufourea) chagrina (Warncke).

\section{2- Dufourea (Dufourea) nodicornis (Warncke 1979)}

\section{Published records from neighboring countries:}

Warncke (1979): 111- 155: Original description and distribution (Morocco, Tunisia, Libya, Israel, Jordon and Syria): Dufoura nodicornis sp. n. Ebmer (1993): Distribution (Tunisia, Jordon and Syria): Dufourea (Dufourea) nodicornis (Warncke). Ebmer (1999): 188: Distribution (Libya, Tunisia, Algaria and Israel): Dufourea (Dufourea) nodicornis (Warncke).

\section{3- Rophites algirus algirus Pérez, 1895: 51-56}

\section{Published records from neighboring countries:}

Ebmer \& Schwammberger (1986), Senckenbergiana Biologica, 66: 277 - 278 (key to females); 278 - 280 (key to males); Distribution (North Africa: Algeria). Type locality: Morocco, Tunisia (new record), Israel and Libanon; Rophites algirus algirus Pérez. 


\section{REFERENCES}

1. Alfken, J. D. 1927. Uber eine Bienenausbeute vonÄgypten. Bull. Soc. Entom. Egypt, 1926: 102 - 106.

2. Baker, D. B. 1996. Notes on some Palearctic and oriental Systropha with descriptions of new species and a key to the species. Journal of Natural History, 30: $1527-1547$.

3. ------------. 2002. On Palearctic and oriental species of the genera Pseudapis W. F. Kirby, 1900, and Nomiapis Cockerell, 1919. Beitrag zur Entomologie, 52: 183.

4. Blüthgen, P. 1925. Die Bienengattung Nomioides Schenck. Stettiner Entomologische Zeitung, 86: 1 - 100.

5. - - 1933. Ein Beitrage zur Kenntnis der Bienenfauna Äegyptens (Hymenoptera: Apidae: Halictidae: Halictinae). Bulletin de la Société Royale Entomologique d'Egypte, (1-3): 14-27.

6. - 1934. Zweiter Beitrage zur Kenntnis der Halictinenfauna Äegyptens (Hymenoptera: Apidae: Halictidae: Halictinae). Bulletin de la Société Royale Entomologique d'Egypte, 18: 188-201.

7. Debski, B. 1917. Sur la femelle Nomioides fasciata Friese et description des auters Nomioides d' Egypt. Bull. Soc. Entom. Egypt, 10: 25 - 50.

8. Ebmer, A.W. 1984. Die westpaläarktischen Arten der gattung Dufourea Lepeletier 1841 mit illustrierten Bestimmungstabellen (Insecta: Hymenoptera: Apoidea: Halictidae: Dufoureinae). Senckenbergiana biol., 64: 313-379.

9. - - 1993. Die westpaläarktischen Arten der Gattung Dufourea Lepeletier 1841 mit illustrierten Bestimmungstabellen (Insecta: Hymenoptera: Apoidea: Halictidae: Rophitinae). Dritter Nachtrag. Linzer biologische Beitrage, 25/1: 1542.

10. - - 1999. Die westpaläarktischen Arten der Gattung Dufourea Lepeletier 1841 (Insecta: Hymenoptera: Apoidea: Halictidae: Rophitinae). Vieter Nachtrag. Linzer biologische Beitrage, 31/1: 183-228.

11. El-Akkad, M. K. 1993. Taxonomical and ecological studies of wild bees of subfamily Halictinae (Order Hymenoptera, family Halictidae) in Egypt. Unpublished Ph. D. Thesis, faculty of Agriculture, Moshtohor, Zagazig University, $225 \mathrm{pp}$.

12. Friese, H. 1898. Beiträge zur Bienenfauna von Aegypten. Természetrajzt Füzetek, 21: 303-313.

13. Handlirsch, A. 1888. Die Bienengattung Nomioides Schenck. Verh. Zool. Bot. Ges. Wien, 38: $395-407$. 
14. Michener, C. D. 2007. The Bees of the World (Second Edition), Baltimore: Johns Hopkins University Press, 953 pp.

15. Pauly, A. 1990. Classification des Nomiinae Africains. Annales Musée Royal de I'Afrique Central, Sciences Zoologiques, 261: 1-206.

16. -------------. 2012. http://zoologie.umh.ac.be/hymenoptera/page.asp?id=72 (Nomiapis);id=82 (Nomia s. str.); id=83 (Pseudapis); Id=96 (Nomioides); id= 94 (Ceylalictus); id=156 (Leuconomia); id=181 (Systropha); id=189 (Rophites); id 191 (Dufourea); id=193 ( Trilia).

17. Pesenko, Yu. A. 1983. Tribe Nomioidini (in the Palearctic fauna), in Fauna of the USSR, Insecta - Hymenoptera, Halictidae, subfamily Halictinae, 17(1): 1 - 198.

18. - 2000. Phylogeny and classification of the family Halictidae revised. (Hymenoptera: Apoidea). J. Kansas Entom. Soc., (1999), 72(1): 104-123.

19. - 2007. The family Halictidae (Hymenoptera): General, In: A key to insects of Russian Far East [In Russian]. Vol. IV, Valdivostok: 745 - 754.

20. Pesenko, Y. \& Pauly, A. 2005. Monograph of the bees of the subfamily Nomioidinae (Hymenoptera: Halictidae) of Africa (excluding Madagascar). Annales de la Société entomologique de France: 41 (2): 129-236.

21. --------. 2009. A contribution to the fauna of the Nomioidine bees of the Arabian Peninsula (Hymenoptera, Halictidae). Fauna of Arabia, 24: 217-236.

22. Saini, M. S. \& Vikram, R. S. 2012. A species checklist of family Halictidae (Hymenoptera: Apoidea) among with keys to subfamilies, genera \& subgenera from India. International Journal of Environmental Sciences, 3 (1): 134 - 166.

23. Shalaby, F. 1958. Alphabetical list of Egyptian Insects in the Collection of the Ministry of Agriculture. Ministry of Agric. Technical Bulletin No. 284, 135 pp.

24. Shoukry, A., M. K. El-akkad, S. M. Kamel and Samia El-akkad 2004. Revision of subfamily Nomiinae of Egypt (Hymenoptera: Halictidae). Agriculture Research Journal, Suez Canal University, 3, (1): 145 - 158.

25. Warncke K. 1976. Zur Systematik und Verbreitung der Bienengattung Nomia Latrielle in der Westpaläarktis und dem turkestanischen Becken. Reichenbachia, 16: $93-120$.

26. - - 1979. Beiträge zur Bienenfauna des Iran: 3. Die Gattung Rophites Spin. mit einer revision des West paläarktischen arten der Bienengattung Rophites Spin. Bollettino del Museo Civico di Storia Naturale di Venezia, 30: 111155.

27. - 1980. Die bienengattungen Nomia und Systropha im Iran mit erganzungen zu den Nomia-Arten der Westpaläarktis. Linzer Biologische Beiträge, 12: $363-384$. 
قائمة تصنيفيه لفصيلة هاليكتيدى في مصر (رتبة غشائية الأجنحة)

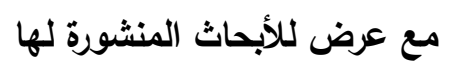

r - تحت فُصيلات نوميويدينى ، نوميينى وروفيتينى لوابد

محمد كمال العقاد وعماد الدين عبد الفتاح معوض بييرس

معهد بحوث وقاية النباتات ، مركز البحوث الزراعية ، الدقى ، الجيزة ، مصر

إثتنملت الورقه الثانية من القائمة التصنيفية على الأنواع المصرية من فصيلة هاليكتيدى (رتبة

غشائية الأجنحة) لتحت فُصيلات نوميويدينى ، نوميينى وروفيتينى مع عرض لجميع الأبحاث المنشورة

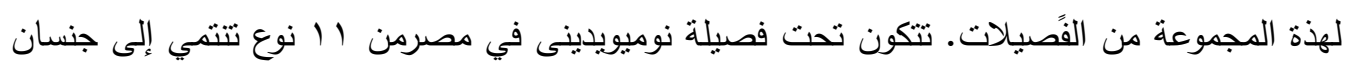

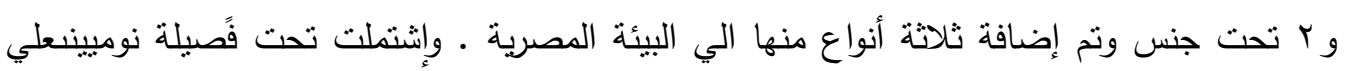

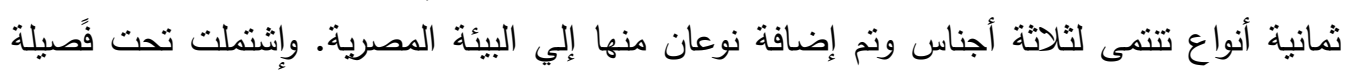

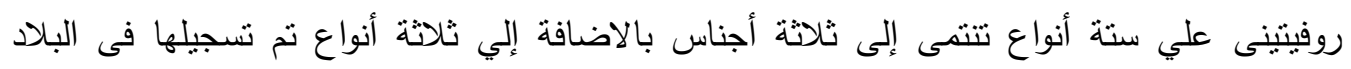

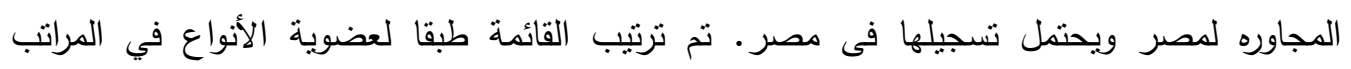
التصنيفية العليا وطبقا لمعظم المراجع التصنيفية الحديثة لفصيلة هاليكتيدى. 